\title{
Role of symmetries in nuclear physics
}

\author{
Venkata Krishna Brahmam Kota ${ }^{1, a}$ and Ashok Kumar Jain ${ }^{2}$ \\ ${ }^{1}$ Physical Research Laboratory, Ahmedabad 380 009, India \\ 2 Amity Institute of Nuclear Science \& Technology, Amity University, Noida 201301, \\ UP, India
}

Received 2 September 2020 / Accepted 2 September 2020

Published online 23 October 2020

\begin{abstract}
We briefly describe the motivation for the special issue on 'Role of symmetries in Nuclear Physics'. In addition, we also present an overview of the topics covered in this special issue.
\end{abstract}

Symmetries pervade every area of physics and are universally accepted to play a unifying role, more so in the microscopic domain. However, nearly all the symmetries, including those having geometric connotations, are invisible. This invisibility imparts them a mysterious nature, manifesting in many mathematical groups. We confirm their presence by various observables in experimental data, like specific patterns in the energy spectra, special relationships between transition rates, and emergence of various selection rules. Heisenberg's introduction of isospin with $S U(2)$ [1,2], Wigner's spin-isospin $S U(4)$ [3], Elliott's $S U(3)$ for rotational nuclei [4-7] and Racah's pairing quasi-spin $S U(2)[2,8]$ algebras are benchmark examples of symmetry principles in nuclei. These various Lie algebraic symmetries and their many extensions within the nuclear shell model $[2,9]$ have been investigated during the nineteen sixties and they played a major role in providing an organized description of nuclear data and a deeper understanding of nuclear structure [10-13].

A renaissance in applying symmetries to organize and explain large amount of nuclear data was witnessed by the introduction of the interacting boson model [14] in the late seventies with $U(6)$ spectrum generating algebra containing vibrational $U(5)$, rotational $S U(3)$, and $\gamma$-unstable $O(6)$ symmetries. Various extensions of the interacting boson model also came into being, which include interacting boson-fermion models for odd mass and odd-odd nuclei developed during eighties and nineties and applications to quantum phase transitions and clustering in nuclei in the last 20 years [15-18]. In addition, a new line of research using point group symmetries for cluster configurations in lighter $N \sim Z$ nuclei has opened up $[19,20]$. All these and extensions of the shell model description to include multi-shell excitations using $S U(3)$ basis and also via the so called $S p(6, R) \supset S U(3)$ symmetry [21-23], giving no-core shell model, established symmetry principles to be an important part of nuclear physics.

Besides these, opening a new direction, there is growing evidence for the importance of isospin and also spin-isospin $S U(4)$ in heavy nuclei [24-26]. On the other hand, there are several new studies involving fundamental symmetries such as rotational invariance, time-reversal, parity and so on in statistical nuclear physics with particular reference to spectral and strength fluctuations, statistical nuclear reactions and spectral averaged quantities such as level densities and strength distributions

a e-mail: vkbkota@prl.res.in 
using random matrix theory $[27,28]$. Continuing with all these exciting developments in nuclear structure physics in particular and nuclear physics in general, many new perspectives in the applications of symmetries in nuclei have emerged in the last 1015 years. The purpose of this special issue is to bring focus to the new developments in this subject. We will now turn to a brief description of the various contributions in this issue.

Role of discrete or point-group symmetries, well known in molecular and solidstate physics, in lighter $N \sim Z$ nuclei such as ${ }^{12} \mathrm{C},{ }^{13} \mathrm{C},{ }^{16} \mathrm{O}$ and so on that exhibit clustering is now receiving new attention with growing experimental data. Santana Valdés and Bijker in their paper [29] present results for configurations generated by double point groups $\mathcal{D}_{3 h}^{\prime}$ and $\mathcal{T}_{d}^{\prime}$ as applicable to $N=Z \pm 1$ nuclei.

Turning to the $S U(3)$ algebra for quadrupole deformed nuclei, there are four contributions. In the first one, Bonatsos et al. [30] showed that it is possible to describe, with proper choice of the ground state $S U(3)$ irreducible representations of various nuclei, within the pseudo- $S U(3)$ model for heavy nuclei, transition from prolate to oblate shape and the prolate over oblate dominance seen in rare-earth nuclei. Sahu et al. [31] in their paper focused on the new paradigm of multiple $S U(3)$ algebras in shell model and interacting boson spaces. In the sdgi space with eight $S U(3)$ algebras, it is shown that six of them generate polate and other two oblate shape. Also, there are situations with rotational structure for yrast levels with weak quadrupole transition strengths among them. In another contribution, Leviatan [32] deals with the novel notion of partial dynamical symmetry (PDS). In a given model space, it is possible to construct Hamiltonians with PDS so that only a subset of states carries a good symmetry but not all the states in the model space. Construction and applications of Hamiltonians with $S U(3)$ PDS, within the interacting boson model, for single and also coexisting quadrupole shapes are reviewed. In addition, some new results for $S U(3)$ PDS in proton-neutron interacting boson model with aligned axially deformed proton-neutron shapes are reported. The fourth contribution is by Kristina et al. [33] and, by expanding their recent work, they establish that ab initio nuclear structure calculations reveal patterns that are consistent with an approximate symplectic $S p(6, R)$ symmetry and seem to emerge from the nature of nuclear interactions. These results have the potential to open new avenues in ab initio no-core shell model calculations of intermediate-mass nuclei.

More complex nuclear shapes like the octupole, tri-axial etc. $[6,12]$ have been part of the frontline research in nuclear physics. Moving to octupole collectivity, Van Isacker [34] has proposed a solvable model in terms of shell model particle-hole excitations for octupole phonons and compared the results of this model with realistic shell model calculations for ${ }^{208} \mathrm{~Pb}$. Besides deformation, pairing is well known to play an important and crucial role in nuclei. Agrawal and Maheswari [35] in their tutorial article, have derived basic results for pairing interaction and the associated quasi-spin and seniority algebra by giving various mathematical details. This article should be very useful for doctoral students and is based on the lectures given by the authors in a school held at Amity University, Noida, India in October 2019. Presenting a new application of the pairing symmetry and related seniority quantum number, Maheswari [36] showed that the multi- $j$ seniority describes reasonably the properties of first excited $2^{+}$and $3^{-}$states observed in $\mathrm{Cd}, \mathrm{Sn}$, and Te isotopes. Going beyond the standard pairing model discussed in $[35,36]$, Feng Pan et al. studied several special types of pairing interactions that can be solved exactly and which have many applications. These include deformed mean-field single particle part, many-body pairing and so on. This work spanning two decades has been meticulously reviewed by Feng Pan et al. [37].

Goodness and usefulness of isospin symmetry in light to medium mass nuclei is well documented. However, in heavy nuclei, where valence protons and neutrons occupy different shells, utility of isospin is still not clear. Swati et al. [38] in their 
article show significantly that there is reasonable empirical evidence for isospin conservation in compound nuclear fusion-fission reactions. Addressing the important issue of symmetry breaking, Cseh [39] discusses general features of dual symmetry breaking, i.e. simultaneous dynamical and spontaneous breaking in various algebraic models. Examples from Elliott's $S U(3)$, interacting boson model, sympletic $S p(6, R)$ model, multi-configurational dynamical symmetry model for cluster configurations and so on are presented.

Unlike the algebraic models, in the commonly used mean-field methods such as Hartree-Fock and Hartree-Fock-Bogoliubov for describing nuclear properties with effective nucleon-nucleon interaction, symmetries such as angular momentum, parity, isospin, particle number etc. are broken. However, in order to compare the results of these methods with the experimental data, symmetry restoration is necessary and methods for these are developed and applied by many groups of researchers. This important topic, with all the technical details, is discussed by Sheikh and Ali [40]. This review article is based on the lectures given by Sheikh in the school held at Amity University, India in October 2019. Final two articles [41,42] are on applications of random matrix theory giving information on symmetries via the statistical energy level fluctuations. Priyanka Rao et al. [41] consider random matrix models with random two-body interactions in the presence of a mean-field one-body part and show that the distribution of higher order spacing ratios gives information about symmetries such as isospin in lighter nuclei and interacting boson model based $F$-spin in heavier nuclei. Similarly, Dwivedi et al. [42] present results for spectral fluctuations in the energy levels of the superdeformed ${ }^{152}$ Dy nucleus possessing different energy levels with different shapes such as axially symmetric quadrupole and octupole shapes. We hope that the articles in this special issue will stimulate further research directions related to symmetries in atomic nuclei and their applications.

In the end, we wish to thank all the authors for their valuable contributions and all the reviewers for providing services to maintain high quality of the manuscripts in this special issue. We hope that the researchers in nuclear physics will find this issue useful for many years to come. Finally, we would like to thank Sabine Lehr, Christan Caron, Vincent Colpin and Sandrine Karpe of EPJST for all the support in completing the task of bringing out the special issue. Our special thanks are due to Prof. B. Ananthanarayan for his keen interest in this project. We feel particularly happy that this issue could see the light of the day in the difficult times of the covid19 pandemic.

\section{References}

1. W. Heisenberg, Z. Phys. 77, 1 (1932)

2. I. Talmi, Simple models of complex nuclei: the shell model and the interacting boson model (Harwood, New York, 1993)

3. E.P. Wigner, Phys. Rev. 51, 106 (1937)

4. J.P. Elliott, Proc. Roy. Soc. (London) A245, 128 (1958)

5. J.P. Elliott, Proc. Roy. Soc. (London) A245, 562 (1958)

6. A. Bohr, B.R. Mottelson, Nuclear structure. Vol II. Nuclear deformations (W.A. Benjamin, Inc., Reading, MA, 1975)

7. V.K.B. Kota, SU(3) symmetry in atomic nuclei (Springer Nature, Singapore, 2020)

8. G. Racah, Phys. Rev 63, 367 (1943)

9. M. Goeppert-Mayer, J.H.D. Jensen, Elementary theory of nuclear shell structure (John Wiley \& Sons, New York, 1955)

10. D.M. Brink et al., Symmetry properties of nuclei, in Proceedings of the 15th Solvay Conference on Physics, September 28-October 3, 1970 (Gordon and Breach, New York, 1974) 
11. A. Frank, J. Jolie, P. Van Isacker, Symmetries in atomic nuclei: from isospin to supersymmetry, 2nd edn. (Springer, New York, 2019)

12. D.J. Rowe, J.L. Wood, Fundamentals of nuclear models (World Scientific, Singapore, 2010)

13. S.C. Pancholi, Pear-shaped nuclei (World Scientific, Singapore, 2010)

14. A. Arima, F. Iachello, Phys. Rev. Lett. 35, 1069 (1975)

15. F. Iachello, A. Arima, The interacting boson model (Cambridge University Press, Cambridge, 1987)

16. F. Iachello, P. Van Isacker, The interacting boson-fermion model (Cambridge University Press, Cambridge, 1991)

17. P. Cejnar, J. Jolie, R.F. Casten, Rev. Mod. Phys. 82, 2155 (2010)

18. V.K.B. Kota, R. Sahu, Structure of medium mass nuclei: deformed shell model and spin-isospin interacting boson model (CRC Press, Taylor \& Francis group, Boca Raton, FL, 2017)

19. D.J. Marín-Lámbarri, R. Bijker, M. Freer, M. Gai, Tz. Kokalova, D.J. Parker, C. Wheldon, Phys. Rev. Lett. 113, 012502/1 (2014)

20. R. Bijker, F. Iachello, Phys. Rev. Lett. 112, 152502/1 (2014)

21. D.J. Rowe, Prog. Part. Nucl. Phys. 37, 265 (1996)

22. K.D. Launey, T. Dytrych, J.P. Draayer, Prog. Part. Nucl. Phys. 89, 101 (2016)

23. T. Dytrych, K.D. Launey, J.P. Draayer, P. Maris, J.P. Vary, E. Saule, U. Catalyurek, M. Sosonkina, D. Langr, M.A. Caprio, Phys. Rev. Lett. 111, 252501/1 (2013)

24. D. Robson, Science 179, 133 (1973), [erratum Science 180, 724 (1973)]

25. S. Garg, B. Maheshwari, A.K. Jain, Phys. Scr. 93, 124008/1 (2018)

26. Yu.S. Lutostansky, V.N. Tikhonov, EPJ Web Conf. 107, 06004/1 (2016)

27. M.L. Mehta, Random matrices, 3rd edn. (Elsevier B.V., Netherlands, 2004)

28. V.K.B. Kota, Embedded random matrix ensembles in quantum physics, Lecture Notes in Physics 884 (Springer, Heidelberg, 2014)

29. A.H. Santana Valdés, R. Bijker, Eur. Phys. J. Special Topics 229, 2353 (2020)

30. D. Bonatsos, A. Martinou, S. Sarantopoulou, I.E. Assimakis, S. Peroulis, N. Minkov, Eur. Phys. J. Special Topics 229, 2367 (2020)

31. R. Sahu, V.K.B. Kota, P.C. Srivastava, Eur. Phys. J. Special Topics 229, 2389 (2020)

32. A. Leviatan, Eur. Phys. J. Special Topics 229, 2405 (2020)

33. K.D. Launey, T. Dytrych, G.H. Sargsyan, R.B. Baker, J.P. Draayer, Eur. Phys. J. Special Topics 229, 2429 (2020)

34. P. Van Isacker, Eur. Phys. J. Special Topics 229, 2443 (2020)

35. B.K. Agrawal, B. Maheshwari, Eur. Phys. J. Special Topics 229, 2459 (2020)

36. B. Maheshwari, Eur. Phys. J. Special Topics 229, 2485 (2020)

37. F. Pan, X. Guan, L.-R. Dai, Yu Zhang, J.P. Draayer, Eur. Phys. J. Special Topics 229, $2497(2020)$

38. S. Garg, A.K. Jain, Y. Sun, A. Goel, Eur. Phys. J. Special Topics 229, 2527 (2020)

39. J. Cseh, Eur. Phys. J. Special Topics 229, 2543 (2020)

40. J.A. Sheikh, R.N. Ali, Eur. Phys. J. Special Topics 229, 2555 (2020)

41. P. Rao, M. Vyas, N.D. Chavda, Eur. Phys. J. Special Topics 229, 2603 (2020)

42. N. Dwivedi, B. Agarwal, S.R. Jain, Eur. Phys. J. Special Topics 229, 2619 (2020) 